\section{The clip-and-snare method with a pre-looping technique during gastric endoscopic submucosal dissection}

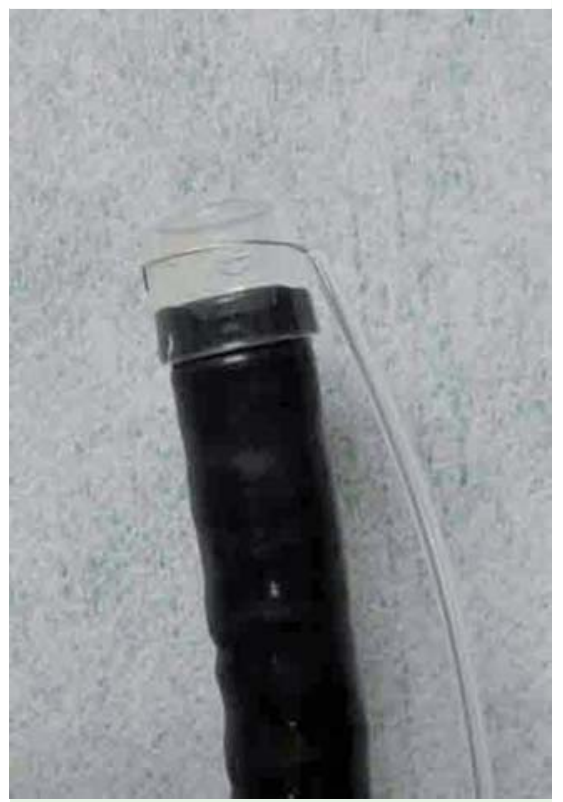

Fig. 1 The transparent cap on the endoscope tip is tightened from outside the endoscope by an assistant using the snare.

The effectiveness of the clip-and-snare method, which is a traction method used during gastric endoscopic submucosal dissection (ESD), has been reported previously $[1,2]$. In previous reports, an additional forceps was required to deliver the snare to an anchored clip. However, this delivery technique is difficult and risks gastric tissue injury, especially when lesions are located in the upper body. In this report, we describe a newly developed pre-looping technique as an easy and safe delivery method.

A 72-year-old man underwent ESD of an upper gastric lesion. A single-channel endoscope (GIF-Q260J; Olympus Medical Systems Corporation, Tokyo, Japan) with a disposable transparent cap (D-201-11804; Olympus) on the endoscope tip was used. A mixture of saline solution, $0.4 \%$ sodium hyaluronate, and indigo carmine was injected into the submucosal layer surrounding the lesion, and a circumferential incision was made with an insulation-tipped scalpel (ITKnife2, Olympus). The endoscope was then withdrawn, and the snare was looped around the transparent cap (SD-221U-25; Olympus) from outside the endoscope ( Fig. 1). The endoscope and snare were reinserted into the lesion. Then, with a reusable clip deployment device (EZ Clip; Olympus), a hemoclip (HX-610-090; Olympus) was inserted through the endoscope channel and used to grasp the aboral side of the lesion ( $\bullet$ Fig.2). The pre-looped snare was then loosened from the transparent cap, with care taken not to release the hemoclip completely from its deployment device. The snare was then moved along the device toward the hemoclip ( Fig.3). The hemoclip was grasped with the snare and released from the deployment device. Then, the appropriate tension was applied to the lesion with the snare, independently of the endoscope, and the submucosal layer was successfully incised ( Fig.4). The 7-mm adenocarcinoma was resected en bloc in 23 minutes without complications.

The pre-looping technique enabled the easy and safe use of the clip-and-snare method in the treatment of an upper gastric lesion ( Video 1 ).

\section{Video 1}

Endoscopic submucosal dissection of an upper gastric lesion is performed with the clip-andsnare method, with pre-looping of the snare around the transparent cap at the tip of the endoscope. The pre-looping technique enables the easy and safe use of the clip-and-snare method in the treatment of an upper gastric lesion.

Endoscopy_UCTN_Code_TTT_1AO_2AG

\section{Competing interests: None}

\section{Naohiro Yoshida, Hisashi Doyama, Ryosuke Ota, Kunihiro Tsuji}

Department of Gastroenterology, Ishikawa Prefectural Central Hospital, Ishikawa, Japan

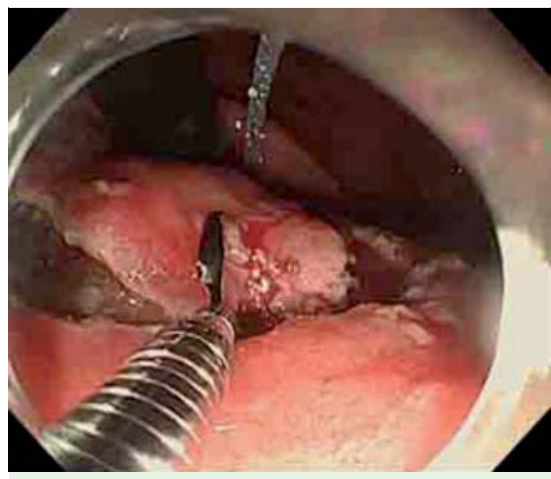

Fig. 2 A reusable clip deployment device and hemoclip are inserted through the endoscope channel. Initially, the lesion is grasped in retroflexion with the hemoclip. It is important not to release the hemoclip completely from the device during this maneuver.

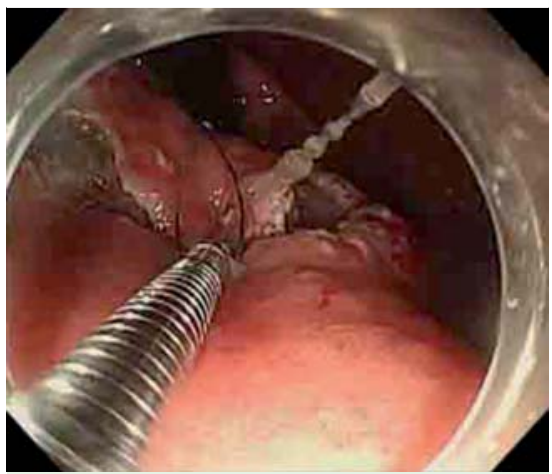

Fig. 3 The lesion has been grasped by the hemoclip. Then the assistant loosens the prelooped snare from the endoscope attachment. The surgeon pulls the snare orally and moves it along the deployment device toward the hemoclip. Then, the assistant uses the snare to grasp the hemoclip, and another assistant releases the hemoclip from the deployment device.

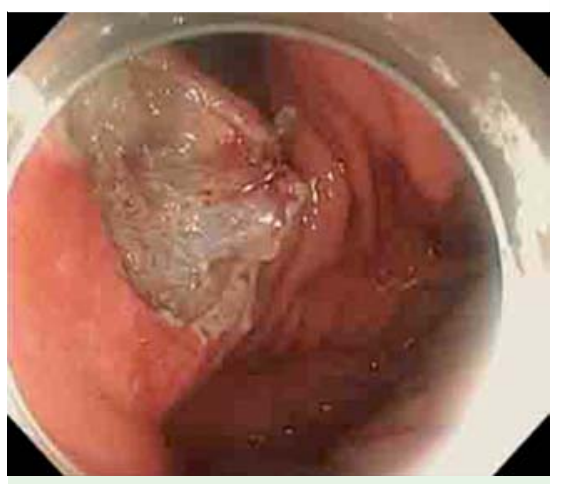

Fig. 4 The surgeon applies the appropriate tension to the lesion by pulling or pushing the snare independently of the endoscope and obtains a good field of view. 


\section{References}

1 Yasuda M, Naito Y, Kokura $S$ et al. Newlydeveloped ESD (CSL-ESD) for early gastric cancer using convenient and low-cost lifting method (lifting method using clips and snares) for lesions is clinically useful [abstract 244]. Gastrointest Endosc 2012; 75 (Suppl. 01): Sa1687

2 Baldaque-Silva F, Vilas-Boas F, Velosa $M$ et al. Endoscopic submucosal dissection of gastric lesions using the "yo-yo technique". Endoscopy 2013; 45: $218-221$

\section{Bibliography}

Dol http://dx.doi.org/

10.1055/s-0034-1390752

Endoscopy 2014; 46: E611-E612

(c) Georg Thieme Verlag KG

Stuttgart · New York

ISSN 0013-726X

\section{Corresponding author}

\section{Naohiro Yoshida, MD}

Department of Gastroenterology Ishikawa Prefectural Central Hospital 2-1 Kuratsukihigashi, Kanazawa Ishikawa 920-8530

Japan

Fax: +81-76-238-2337

ynaohiro@ipch.jp 